\title{
Mini-Review
}

Theme: Clinical and Commercial Translation of Drug Delivery Systems

Guest Editors: Yoon Yeo and Craig Svensson

\section{Drug Carriers: Not an Innocent Delivery Man}

\author{
Yoon Yeo ${ }^{1,2,3,4}$ and Bieong-Kil Kim ${ }^{1}$
}

Received 31 March 2015; accepted 12 May 2015; published online 28 May 2015

Abstract. Biomaterials used as drug carriers are often considered inactive and assumed to have no other roles than modifying pharmacokinetics and biodistribution of a drug. On the other hand, there are several examples in which the carrier materials show bioactivities in the body, which may have been underestimated or inadvertently ignored. This review highlights several examples where biomaterials used as drug carriers bring biological effects, known or newly discovered, and discusses their implications in development of new drug delivery systems.

KEY WORDS: biological effects; biomaterials; drug carriers; drug delivery; inactive ingredients.

\section{INTRODUCTION}

One of the primary requirements of a drug carrier is biocompatibility-the lack of tissue reactions against the material causing premature removal and/or unwanted immune responses. Various natural and synthetic polymers have been explored as biocompatible drug carriers, under an assumption that they have no other roles than delivering the payload to target tissues. However, many studies including ours show that the carriers may play more active roles in therapeutic effects of the product, although some of them may not have been explicitly discussed as the main topic of the study. For example, we have reported that a new chitosan derivative has a unique ability to suppress pro-inflammatory cytokine production from endotoxin-challenged macrophages (1). Moreover, we have delivered platinum into the peritoneal cavity using hyaluronic acid nanoparticles and hydrogels for local chemotherapy of ovarian cancer and found that they rather cause a slight increase in tumor burdens at later time points (2), which suggests a potential involvement of empty carriers and degradation products in the growth of residual tumors. These studies indicate that drug carriers can have biological effects to the host, which may not have been anticipated during the system design or inadvertently ignored in data analysis. The potential effect of biomaterial itself is a

\footnotetext{
${ }^{1}$ Department of Industrial and Physical Pharmacy, Purdue University, 575 Stadium Mall Drive, West Lafayette, Indiana 47907, USA.

${ }^{2}$ Weldon School of Biomedical Engineering, Purdue University, West Lafayette, Indiana 47907, USA.

${ }^{3}$ Biomedical Research Institute, Korea Institute of Science and Technology, Hwarangno 14-gil 5, Seongbuk-gu, Seoul, 136-791, South Korea.

${ }^{4}$ To whom correspondence should be addressed. (e-mail: yyeo@purdue.edu)
}

significant issue in evaluating new formulations, since the obtained pharmacological activities may not necessarily be due to the alternation of pharmacokinetics/biodistribution alone. Without this knowledge, one may overestimate the functionality of a drug carrier and reach a misleading prediction of clinical effects of the product. In this review, we discuss bioactivities of pharmaceutical ingredients commonly pursued as drug carriers, known or newly discovered, and their implications in drug product development.

\section{CHITOSAN AND CHITOSAN DERIVATIVES}

Chitosan is an aminopolysaccharide composed of Dglucosamine and N-acetyl-D-glucosamine, obtained by partial deacetylation of chitin, a key component of crustacean shells (3). The amine groups in chitosan provide unique features useful for drug delivery, such as $\mathrm{pH}$-sensitive water solubility (soluble in acidic $\mathrm{pH}$, insoluble in neutral or basic $\mathrm{pH}$ ), cationic charges amenable to complexation with anionic macromolecules such proteins and nucleic acids, and chemical reactivity with other functional molecules (4). Therefore, chitosan has been employed in a variety of drug delivery systems from hydrogels to nanoparticles as a standalone carrier or a partial component of a system (5). Another attractive feature of chitosan is the biocompatibility, with negligible toxicity after single or repeated use in oral and parenteral applications $(5,6)$, and the biodegradability based on hydrolysis by enzymes ubiquitous in human body such as lysozyme, lipase, or amylase (3).

As a pharmaceutical excipient, chitosan is often assumed to be a pharmacologically "inert" material, but its biological activities have long been a significant research topic. For example, chitosan is shown to have hemostatic activities and has been pursued as a wound healing agent. The hemostatic activities are independent 
of the classical coagulation cascade but based on charge interactions between cationic chitosan and anionic cell membrane of erythrocytes, which lead to platelet activation and thrombus formation (7). Moreover, chitosan promotes wound healing process by activating macrophages, stimulating cytokine production, and formation of scar tissues $(8,9)$. In addition, chitosan shows antimicrobial activities against a broad spectrum of bacteria with little toxicity toward mammalian cells (10). The mechanism of antimicrobial activities of chitosan remains elusive, although it is frequently attributed to strong electrostatic interactions between chitosan and bacterial cell surface, followed by disruption of membrane barrier functions and leakage of cellular components (10).

The cationic charge of chitosan is also exploited for removing endotoxin, the main cause of bacterial sepsis. Endotoxin has strong negative charges due to lipid A and interacts with chitosan via electrostatic interaction. Machado et al. reported that endotoxin binds to chitosan with $\mathrm{K}_{\mathrm{d}}$ of $10^{-11} \mathrm{M}$ at $\mathrm{pH} 7$, comparable to adsorbents based on polylysine $\left(\mathrm{K}_{\mathrm{d}} 5.5 \times 10^{-11} \mathrm{M}\right)$ or N,N-dimethylaminopropylacrylamide $\left(\mathrm{K}_{\mathrm{d}}\right.$ $\left.7.2 \times 10^{-11} \mathrm{M}\right)$ (11). Chitosan immobilized on a cellulose membrane was shown to remove endotoxin from distilled water and interferon preparations with removal efficiency close to $90 \%$ at optimal conditions (12). However, the poor water solubility of chitosan at physiological $\mathrm{pH}$ limits its utility as a therapeutic agent for systemic treatment of sepsis. In this regard, it is worthwhile to note a water-soluble chitosan derivative created by partial amidation of chitosan (13), called zwitterionic chitosan (ZWC), negatively charged and hence water-soluble at neutral $\mathrm{pH}$ unlike the parent chitosan. Due to the water solubility, intraperitoneally injected ZWC was well absorbed leaving no residues in the peritoneal cavity, whereas a salt form of chitosan (chitosan glutamate) created extracellular deposits, surrounded by large activated macrophages (Fig. 1a) $(1,13)$. Interestingly, ZWC suppressed the production of proinflammatory cytokines by lipopolysaccharide (LPS)-challenged macrophages (Fig. 1b) (1). Moreover, IP-administered ZWC attenuated the onset of endotoxin-induced sepsis in mice, with no signs of adverse tissue responses seen with chitosan (unpublished data). These properties suggest that ZWC may have potential utility in systemic treatment of sepsis.

Biological activity of chitosan derivative was also observed in applications where chitosan was used as a carrier of anti-cancer drugs. Glycol chitosan was modified with $5 \beta$ cholanic acid to form nano-sized self-assemblies consisting of hydrophobic core encapsulating poorly water-soluble drugs and hydrophilic glycol surface (14). The hydrophobically modified glycol chitosan (HGC) nanoparticles were used for the delivery of hydrophobic anti-cancer drugs such as paclitaxel (14), docetaxel (15), or camptothecin (16). The HGC-encapsulated drugs were comparable (14) or superior $(15,16)$ to free drug controls in delaying tumor progression with relatively low systemic toxicity profiles. Interestingly, empty HGC nanoparticles administered at a dose $(200 \mathrm{mg} / \mathrm{kg})$ equivalent to HGC-drug particles at $20 \mathrm{mg} / \mathrm{kg}$ PTX resulted in tumor regression, although the mechanism remained unclear (Fig. 2) (14). The authors speculated that HGC might have changed the local environment of tumor tissues (14).

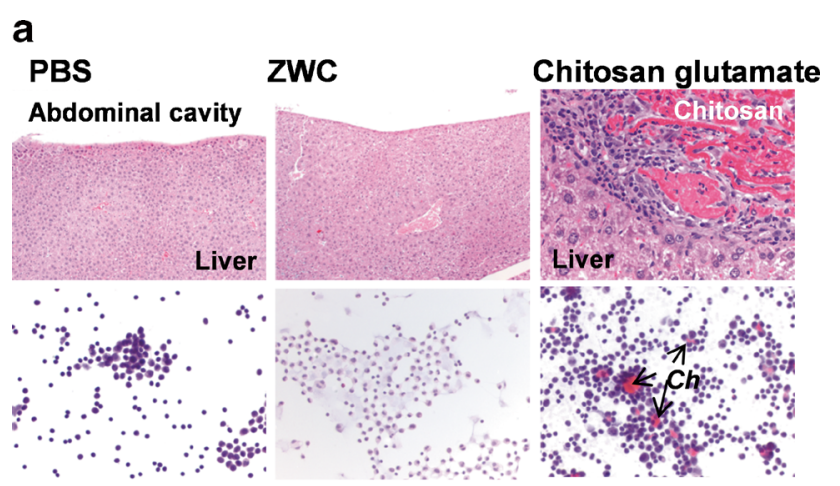

\section{b MIP-2 (pg/mL) TNF- $\alpha(\mathrm{pg} / \mathrm{mL}) \quad$ IL-6 (pg/mL)}

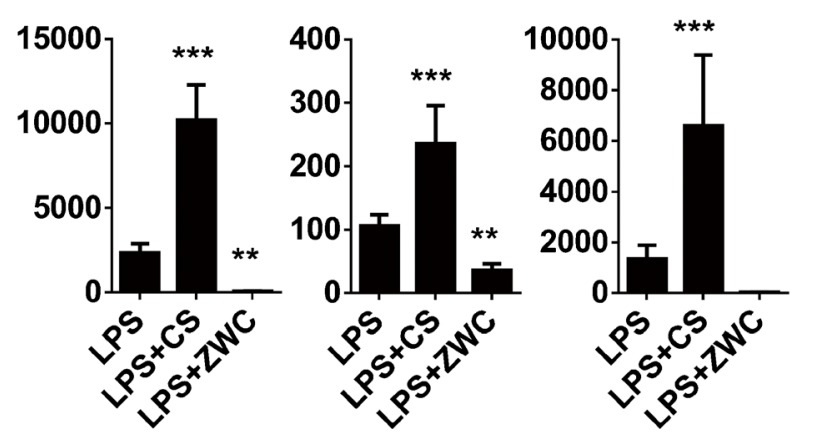

Fig. 1. a (Top) Hematoxylin and eosin (H\&E) staining of liver sections of different treatment groups. (Bottom) Cytology of the peritoneal fluid from different treatment groups using H\&E staining. Ch: extracellular chitosan deposits. b Effect of chitosan treatment (all in $2 \mathrm{mg} / \mathrm{mL})$ on cytokine release from LPS $(1 \mu \mathrm{g} / \mathrm{mL})$-challenged macrophages. $* * p<0.01 ; * * * p<0.001 v s$. LPS. LPS lipopolysaccharide, $C S$ chitosan, $Z W C$ zwitterionic chitosan. Adapted from (1)

\section{HYALURONIC ACID}

Hyaluronan, or hyaluronic acid (HA), is a polysaccharide composed of D-glucuronic acid and N-acetyl-Dglucosamine (17), a glycosaminoglycan abundant in the skin, lung, synovial fluid, and blood (18). HA binds to cluster determinant 44 (CD44) (19), a cell surface protein upregulated in many cancers of epithelial origin (20). Due to this property, HA has been incorporated into various nanomedicine constructs as a targeting ligand $(20,21)$. As a natural component of extracellular matrix tissues, HA is also biocompatible and biodegradable, making an excellent building block for engineered tissue scaffolds and drug delivery systems (22). HA has several biological functions such as lubrication, water homeostasis, macromolecular filtering, and regulation of cellular activities (23). HA is known to activate cell migration and proliferation and regulate inflammation and tissue remodeling, which have been explicitly exploited in tissue engineering and wound healing (24). However, the biological effects of HA on target tissues as drug carriers have not been a topic of active discussion.

We recently observed that HA could bring about undesirable consequences to cancer therapy. We used an in situ crosslinked HA hydrogel as a carrier of platinum in an animal model of intraperitoneal cancer (2). Platinum was first 


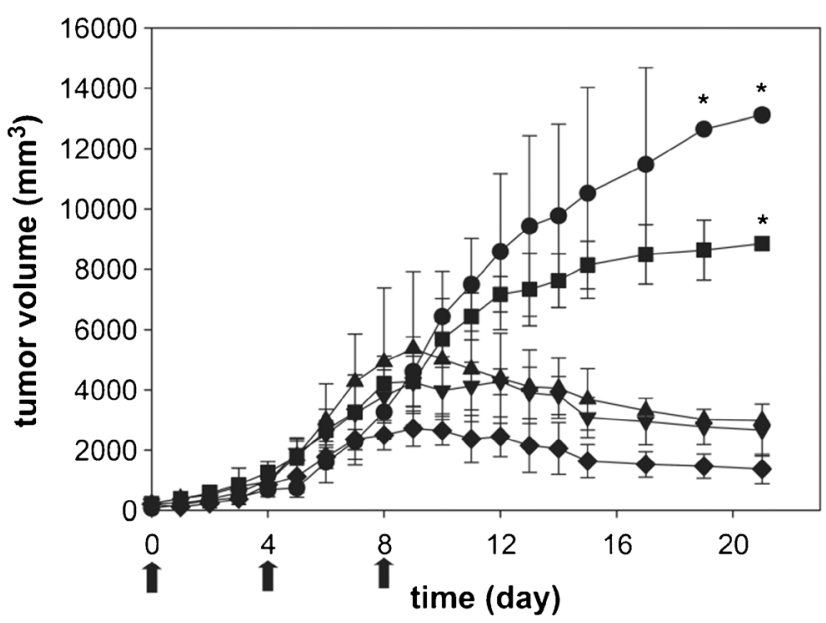

Fig. 2. In vivo anti-tumor activity of HGC, Cremophor EL-based PTX, and PTX-HGC nanoaggregates after administration i.v. Arrows indicate the times of injection The results represent the means $\pm \mathrm{SD}(n=6)$. The symbols are as follows: saline (black circle); HGC (black square); Cremophor EL-based PTX, equivalent to $20 \mathrm{mg} / \mathrm{kg}$ PTX (black triangle); PTX-HGC, equivalent to $20 \mathrm{mg} / \mathrm{kg}$ PTX (black inverted triangle); and PTX-HGC, equivalent to $50 \mathrm{mg} / \mathrm{kg}$ PTX (black diamond). Reprinted with permission from (14). Copyright (C) 2006 Elsevier

encapsulated in HA nanoparticles, mixed in the HA hydrogel precursors, and injected to the peritoneal cavity of animals bearing intraperitoneal tumors to prolong the drug exposure to the tumors. The platinum-loaded HA hydrogel suppressed tumor progression initially to a level comparable to cisplatin (free drug solution) but caused a slight increase in tumor burdens at later time points (4 weeks or later), contrary to our expectation. This observation was not explained by the attenuation of drug release from the HA gel, since the control group administered with two half doses of cisplatin solution with a week interval (mimicking sustained drug release) showed superior tumor regression compared to the platinum-HA gel-treated group. Given that biological activities of HA gel have been exploited in tissue engineering to support the growth of tissues (25), we suspect that the HA gel, after exhausting platinum, may have partaken in the tumor growth (2). In this regard, it is worthwhile to revisit an earlier study reporting the enhancement of intraperitoneal tumor growth in animals treated with HA (26). In addition, it was argued that HA had the potential to interfere with interactions between tumor cells and monocytes or tumorinfiltrating macrophages, which would otherwise have provided anti-tumor effects through their effector functions $(27,28)$. These observations collectively suggest that the empty HA carriers may have cell-proliferative effects, which favor progression of tumors. On the other hand, we do not necessarily preclude the use of HA gel as a drug carrier, especially when the drug can last longer with HA gel, leaving a shorter (or no) period for exposure to drug-free carriers. Studies using paclitaxel and HA gel mixture (29) or covalent conjugates of paclitaxel-HA $(30,31)$, which released drug over a longer period of time than in our platinum study, took advantage of HA's biocompatibility and affinity for CD44 and demonstrated improved safety profiles with comparable or superior anti-tumor effects to Taxol.

\section{CHONDROITIN SULFATE}

Chondroitin sulfate is a polysaccharide consisting of two alternating monosaccharides ( $\mathrm{N}$-acetylgalactosamine and glucuronic acid), present on the cell surface and in the extracellular matrix (32) and covalently bound to proteins to form a proteoglycans (33). Chondroitin sulfate has been widely used in drug delivery as a hydrophilic component of self-assembled nanocarriers (33-39). In addition, due to the anionic charge and the ability to bind to CD44, chondroitin sulfate has been used in combination with polymeric gene carriers to reduce the cationic charge (40-46) and improve the target specificity (45-47). The chondroitin sulfate associated with cationic gene carriers is also shown to modify intracellular trafficking of the gene complex (promote endosomal escape and accumulation at the nuclear periphery) in favor of gene transfection (48).

While most studies have focused on reducing cytotoxicity of polycations with chondroitin sulfate, we recently observed the opposite trend with a chondroitin sulfate-polycation complex. We found that a combination of polyethyleneimine derivative with disulfide crosslinking (49) and chondroitin sulfate-B (also known as dermatan sulfate) showed unique toxicity at a specific weight ratio in a group of cancer cells including B16-F10 melanoma and PC-3 prostate cancer cell lines (unpublished data). Neither dermatan sulfate nor polyethyleneimine derivative showed significant toxicity when used alone. The unique toxicity translated to decrease in tumor density in a mouse model of B16-F10 melanoma (Fig. 3). We speculate that the effect of chondroitin sulfate-polycation complex on a certain group of cancer cells may
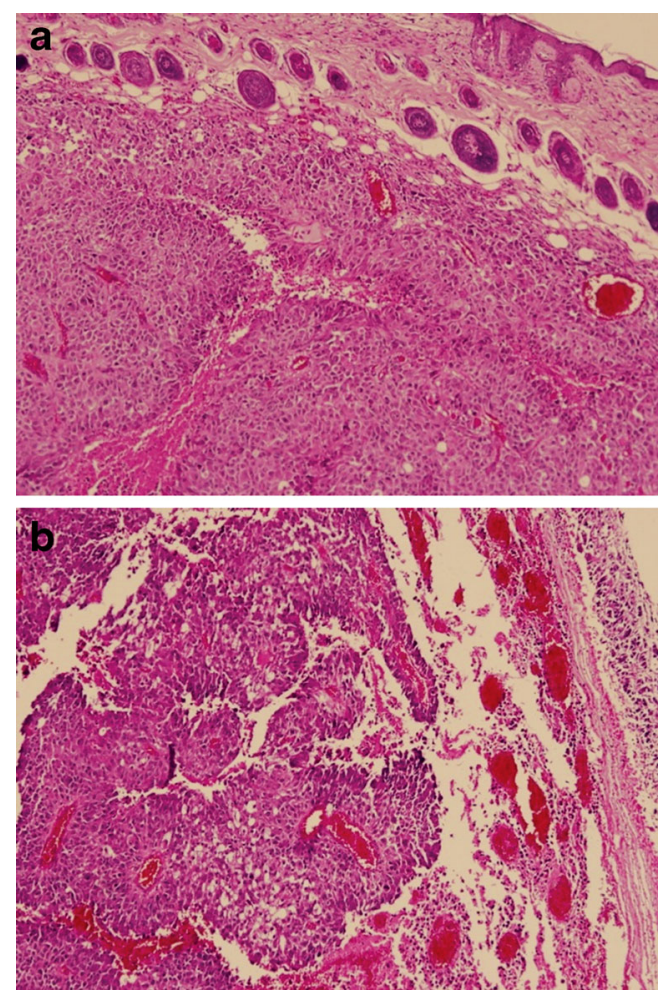

Fig. 3. $\mathrm{H} \& \mathrm{E}$ stained section of B16-F10 tumors in Balb/c mice receiving a no treatment or $\mathbf{b}$ IV injection of a polyethyleneimine derivativedermatan sulfate binary complex 
be due in part to the interference of critical functions of dermatan sulfate in their progression (50-52).

\section{PLURONICS}

Pluronics (also known as Poloxamers) are tri-block copolymers consisting of hydrophilic ethylene oxide (EO) and hydrophobic propylene oxide (PO) blocks, arranged in the order of EO-PO-EO blocks (Fig. 4a) (53). Their amphiphilic structures allow them to serve as a surfactant, forming polymeric micelles to help solubilize hydrophobic compounds. At body temperature, Pluronics have a critical micelle concentration ranging from $1 \mu \mathrm{M}$ to $1 \mathrm{mM}$, depending on the length of EO and PO blocks (53). Kabanov et al. observed that Pluronics sensitized drug-resistant cancer cell lines to anti-cancer drugs, which would otherwise have a limited cytotoxic activity to the cells and confirmed the effect in several drug-resistant tumor models (54). Several mechanisms account for the ability of Pluronics to sensitize drugresistant tumors (Fig. 4b) $(55,56)$. First, Pluronics interfere with drug efflux from the multi-drug-resistant cells and increase intracellular accumulation of anti-cancer drugs. The polymer binds to lipid bilayers, perturbing the structure of cell membranes. This leads to the inhibition of ATPase activity of drug efflux transporters such as Pgp and BCRP. Pluronics also inhibit mitochondrial respiration and ATP synthesis, depleting intracellular energy needed to sustain the drug resistance. Moreover, the interaction of Pluronics and mitochondria leads to production of reactive oxygen species and release of cytochrome c, enhancing drug-induced apoptosis and further contributing to chemosensitization (57). Taking advantage of this unique property, a micelle formulation of doxorubicin incorporated in two Pluronics (SP1049C) was developed and tested in clinical studies (58-60). In a phase 2 clinical study in patients with advanced adenocarcinoma of the esophagus and gastroesophageal junction, SP1049C demonstrated appreciable anti-tumor activity as a single agent and an acceptable safety profile (59). SP1049C is currently pursued as an agent targeting cancer stem cells, which are highly tumorigenic and intrinsically drug resistant $(55,61)$.

\section{POLAR LIPIDS}

Polar lipids are a class of biomolecules with polar head groups linked to hydrophobic tails, classified as phospho-, glyco-, and sulfo-lipids according to the type of polar groups. Due to the amphiphilic nature, polar lipids form nanometer scale assemblies like micelles and liposomes with well-defined biophysical properties. In particular, liposomes have widely been used as carriers for drugs with unfavorable toxicity profiles such as doxorubicin and amphotericin, making the first generation nanomedicines approved by the FDA.

Due to the similarity to mammalian cell membranes and the natural origin of the components, liposomes are considered generally safe and biocompatible (62). However, studies in the past decade report immunomodulatory effects of liposomes. Several components of liposomes account for the immunogenicity. Polyethylene glycol (PEG), added to polar head groups of phospholipids to sterically stabilize liposomes, was found to be responsible for rapid elimination of a second dose of PEGylated liposomes (63). It was later determined that IgM with high affinity for PEG on the liposomes mediated the complement activation, resulting in accelerated clearance and enhanced hepatic uptake of the second-injected PEGylated liposomes (Fig. 5a) (64). In addition, polar lipids with cationic head groups, such as 1,2-dioleoyl-3trimethylammonium-propane (DOTAP), have immunostimulatory properties, activating cellular signaling pathways responsible for production of chemokines and pro-inflammatory cytokines (Fig. 5b) (65). Conversely, cationic lipids also inhibit LPS-induced pro-inflammatory responses via electrostatic interaction with LPS (66) and/ or interfering with TLR-4/MD-2 signaling (67). On the other hand, liposomes containing negatively charged

a
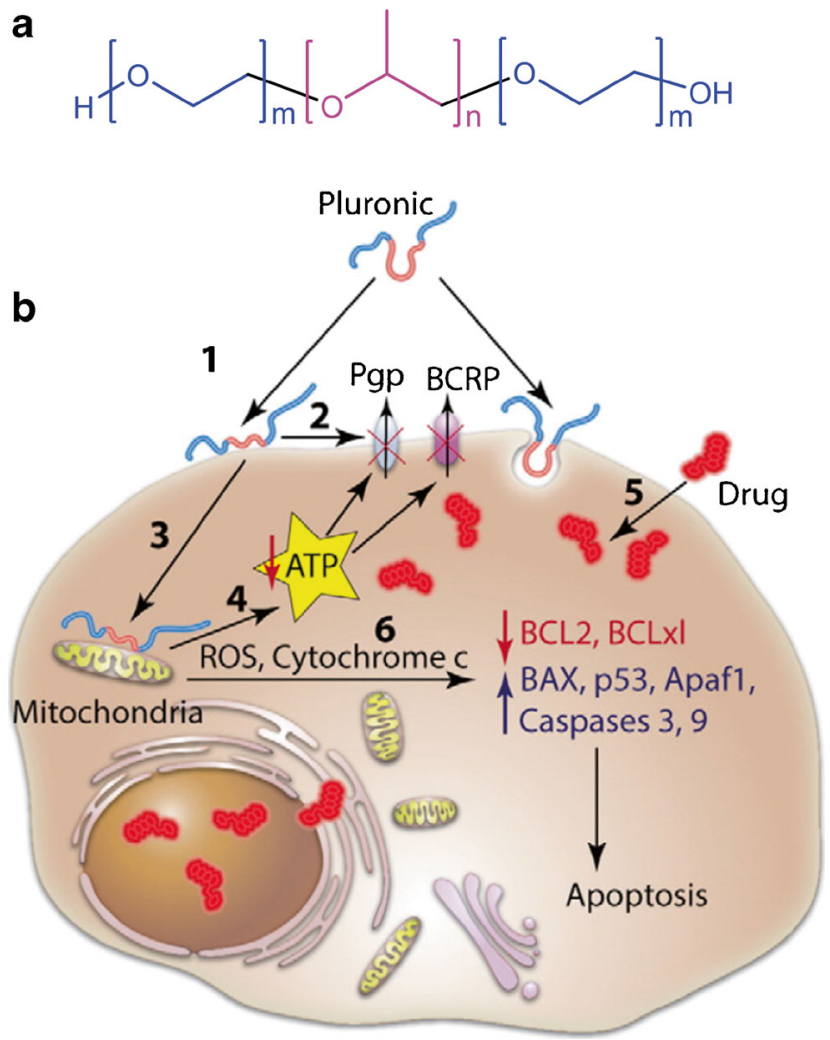

Fig. 4. a Structure of poloxamers. b Schematic representation of multiple effects of poloxamers in MDR cells. Poloxamer molecule binds with the cholesterol-rich domains in the cell membranes ("lipidrafts") and perturbs their structure (1). This results in inhibition of the ATPase activity of the drug efflux pumps, Pgp and BCRP (2). Poloxamer translocates into mitochondria, decreases mitochondria membrane potential and inhibits respiration (3). This leads to inhibition of the mitochondrial H+-ATPase and ATP depletion (4). The ATP depletion (4) along with inhibition of the ATPase activity of the Pgp and BCRP (2) results in the impairment of the drug efflux and increased accumulation of the drug in cells (5). The interaction of poloxamer in mitochondria also releases cytochrome $\mathrm{C}$, increases ROS production, and shifts the cell signaling in response to the drug toward apoptosis (6). Reprinted with permission from (55). Copyright (C) 2013 Elsevier 
Fig. 5. a Accelerated blood clearance of PEGylated liposomes. Rats were pretreated with PEGylated liposomes (lipid dose $0.001 \mu \mathrm{mol} /$ $\mathrm{kg}$ ), followed by second dose of radio-labeled PEGylated liposomes $(5 \mu \mathrm{mol} / \mathrm{kg})$ after different time intervals. Reprinted with permission from (64) Copyright () 2006 Elsevier. b Schematic representation of cell activation by cationic liposomes. I: Cationic liposomes recognize a G-coupled receptor, activate MAP kinases ERK1/2 and p38 MAPK through PI-3 kinase and induce the expression of co-stimulatory molecules (CD80 and CD86) and chemokines (MIP1 $\alpha$, MIP1 $\beta$, MCP1 ) in dendritic cells. II: Cationic liposomes stimulate dendritic cells through TLR4 receptor and activate MAPKs and NF-kB, leading to expression and/or secretion of several chemokines and cytokines (IL12, IL-6, TNF- $\alpha$ ) in dendritic cells. Adapted from ref (65). c Schematic of phosphatidylserine-containing liposomes (PSL) induced pathway for PEG2 production in microglia/macrophages. SR-BI dependent PSL phagocytosis induced ERK1/2 activation to upregulate PEG2 through COX-1/mPGES-2 cascades. The PSL-induced PGE2 suppresses the phagocyte activation. $S R$ - BI class B scavenger receptor type I; ERK1/2 extracellular signal-regulated kinases 1/2; $C O X$ cyclooxygenase; $P G$ prostaglandin; $c P G E S$ cytosolic PGE synthase; $m P G E S$ microsomal PGE synthase. Adapted from (68)

phospholipids, such as phosphatidylserine, mimic the effect of apoptotic cells on phagocytes such as macrophages, microglia, and dendritic cells and cause them to secret anti-inflammatory mediators including prostaglandin E2 (PEG2) and shift from pro- to anti-inflammatory phenotype (Fig. 5c) (68). These immunomodulatory effects of liposomes are now actively pursued as a way of increasing adjuvanicity of vaccines (69) or attenuating inflammation (70-72).

\section{CYCLODEXTRINS}

Cyclodextrins (CDs) are cyclic oligosaccharides composed of six, seven, or eight glucopyranose units, bound via $\alpha$ 1,4-glycosidic linkages, corresponding to $\alpha-\beta$-, and $\gamma$-CD, respectively (73). CDs have a truncated cone structure with a hydrophilic exterior and a hydrophobic interior, which allows them to host hydrophobic small molecular weight drugs and helps solubilize them in aqueous solutions and improve their bioavailability $(74,75)$. For this reason, CDs and their derivatives are widely used as inactive ingredients in more than 35 pharmaceutical products, with well-established monographs in United States Pharmacopeia and National Formulary, Japanese Pharmaceutical Codex, and European Pharmacopoeia (76).

Orally administered CDs are generally considered safe due to the low absorption from the gastrointestinal tract (76). On the other hand, parenteral injection of CDs can cause renal toxicity; hence, only $\alpha-C D$ is used in parenteral products at very low concentrations (76). Recent studies also point to potential cytotoxicity of CDs due to depletion of cell membrane lipids. There is a good correlation between the ability of CDs to solubilize cholesterol, hemolytic activity, and cytotoxicity (77). Cholesterol depletion leads to alteration of several cell functions, including cell morphology, actin cytoskeletal organization, and compositions of cellular proteins and membrane fatty acids (78). In addition to membrane damage, high levels of $\alpha$ - and $\beta$-CDs (0.5 and 1\%) induced apoptosis by activating caspase-3 and caspase-7 (79).

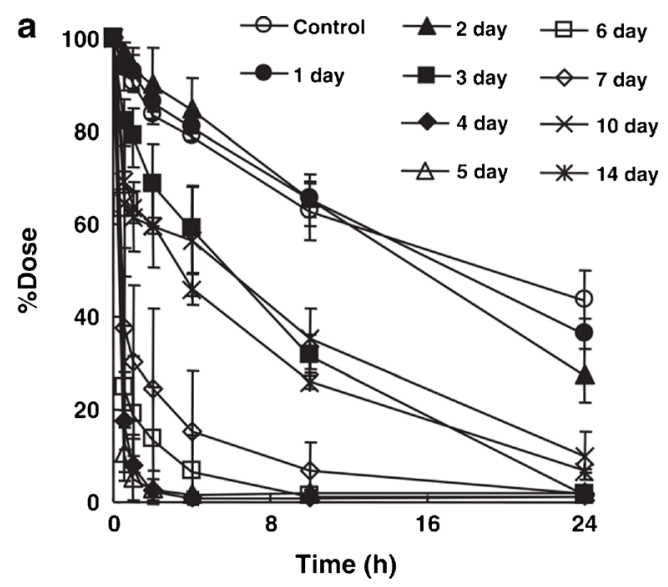

b
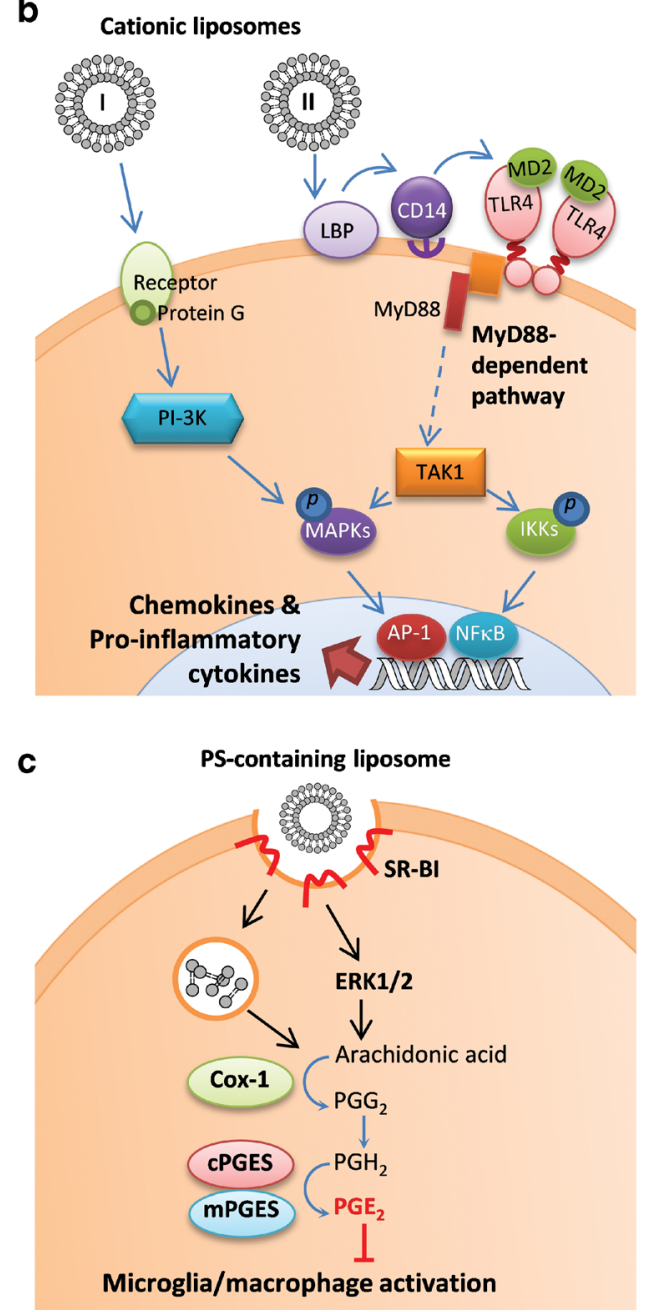

The opposite effects are also reported. Qin et al. reported that the treatment with $10 \mu \mathrm{g} / \mathrm{mL}$ of cholesterol:methyl- $\beta$-CD induced proliferation of primary rat vascular smooth muscle cells by stimulating ERK1/2 signaling (80). Cholesterol depletion by $2 \mathrm{mM}$ of methyl- $\beta$-CD enhanced cell proliferation of primary chick myogenic cells by activation of the Wnt/ $\beta$-catenin pathway (81). While the toxicity of CDs may not be generalized and should be interpreted in the context of 


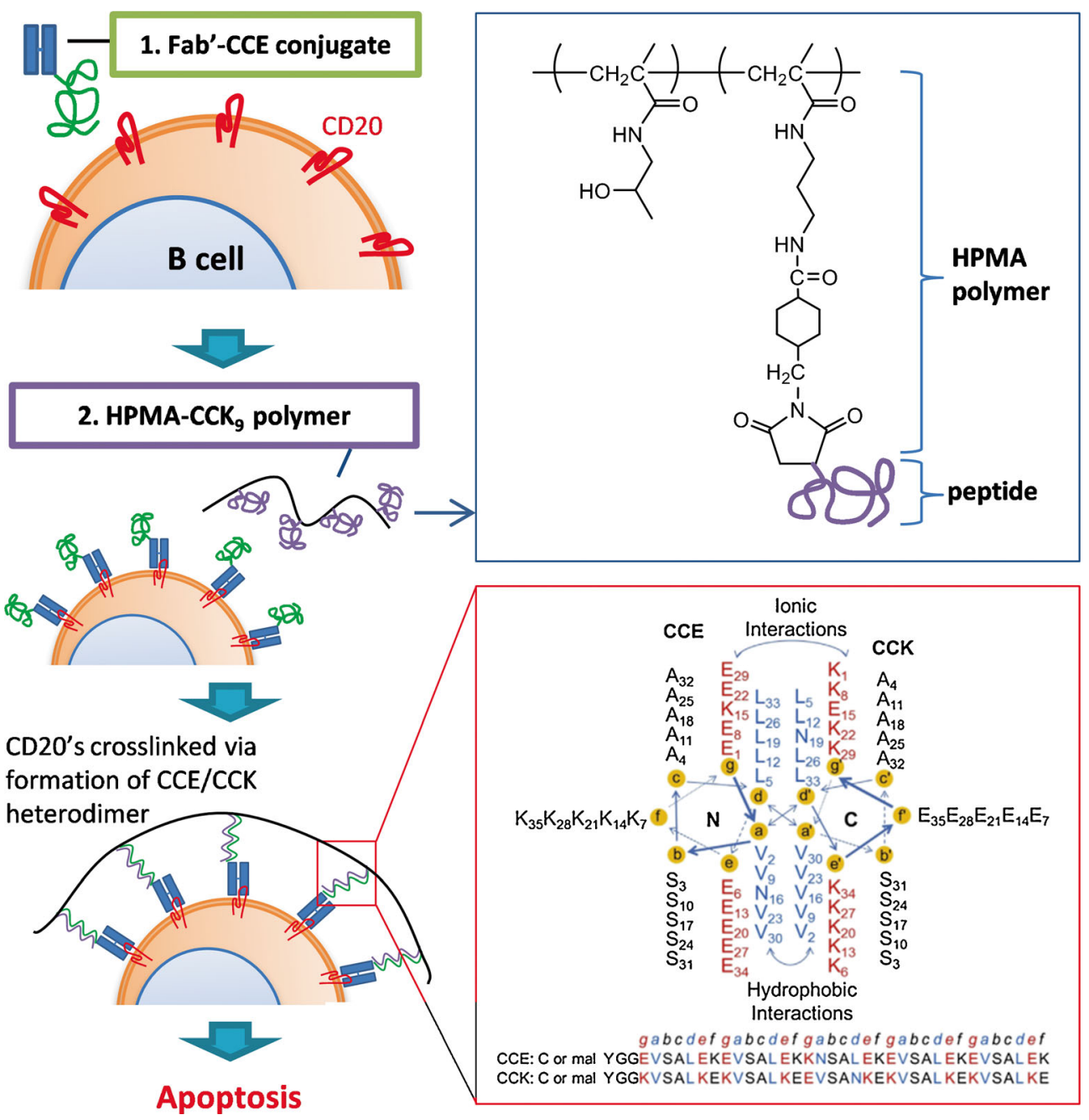

Fig. 6. Schematic of design and mechanism of treatment of NHL with sequential treatment of NHL B cells with Fab '-CCE and HPMA-(CCK)n polymers (adapted from ref. 87). Induction of apoptosis of NHL B cells is triggered by crosslinking of its CD20 antigens mediated by antiparallel coiled-coil CCE/CCK heterodimer formation at the cell surface. Fab'-CCE is a conjugate of the Fab $^{\prime}$ fragment binding to CD20 and the CCE peptide (YGGEVSALEKEVSALEKKNSALEKEVSALEKEVSALEK); HPMA-CCK ${ }_{9}$ is a HPMA copolymer containing 9 grafts of the CCK peptide (CYGGKVSALKEKVSALKEEVSANKEKVSALKEKVSALKE) per macromolecule. CCE/CCK heterodimeric coiled-coil structure was reprinted with permission from (88) Copyright (c) 2014 Elsevier. The view is shown looking down the superhelical axis from the N-terminus of CCE and from the Cterminus of CCK. CC denotes coiled-coil peptides, $\mathrm{E}$ and $\mathrm{K}$ denote peptides in which most of $\mathrm{e}$ and $\mathrm{g}$ positions are occupied by either glutamic acid or lysine, respectively (84)

concentration, their effects on cell membrane lipid content need to be taken into consideration in designing CDcontaining formulations.

\section{N-(2-HYDROXYPROPYL) MATHACRYLAMIDE COPOLYMER ASSEMLIES}

N-(2-hydroxypropyl) methacrylamide (HPMA) copolymers (Fig. 6) are hydrophilic synthetic polymers, developed in 1970s as carriers of anti-cancer drugs and proteins (82). Drug molecules are covalently conjugated as pendants to the HPMA backbone via a linker, which can be cleaved by lysosomal enzymes upon cellular uptake (82). Drug-HPMA conjugates are used to increase the bioavailability and passive accumulation of drugs in tumors and to modify the mechanisms by which drugs are taken up by cells and trafficked in the cells (82). HPMA polymers have been used as a carrier of several anti-cancer drugs including doxorubicin, camptothecin, paclitaxel, and platinates and tested in clinical studies for therapeutic validation $(82,83)$.

More recently, HPMA polymers have been proposed as drug-free macromolecular therapeutics for the treatment of B cell non-Hodgkin lymphoma (NHL) $(84,85)$. This approach is based on the principal that crosslinking of CD20 antigens, a biomarker expressed by most malignant B cells, causes cell apoptosis (86). Here, CD20's on malignant B cells are 


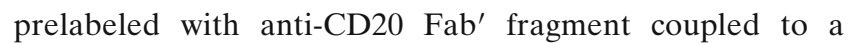
peptide motif (e.g., CCE), which is recognized by a complementary peptide (e.g., CCK) to form an antiparallel coiledcoil heterodimer. The treatment of prelabeled B cells with conjugates of HPMA and multiple grafts of CCK (HPMA$\mathrm{CCK}_{n}$ ) induces crosslinking of CD20 antigens on the cell surface, triggering apoptotic cell death (Fig. 6) (86). Sequential intravenous injections of $\mathrm{Fab}^{\prime}$-CCE and HPMA-CCK ${ }_{n}$ led to extension of the survival period or increase in survival rate in SCID mice bearing human B-lymphoma xenografts (87). As an alternative to peptide pairs, which were found immunogenic in immunocompetent Balb/c mice (88), a complementary pair of morpholino oligonucleotides is currently pursued to improve binding kinetics and efficiency $(89,90)$. The HPMA-based drug-free macromolecular therapeutics shows an example of new roles that the polymers can play beyond their traditional functions as a drug carrier.

\section{FUTURE PERSPECTIVES}

This short review highlights selected examples where biomaterials used as drug carriers show biological effects that may enhance or compromise the pharmacological activities of the payload. Many carrier materials (also called "inactive" ingredients) have been considered biocompatible and often biologically inert. However, the safety and biocompatibility are relative terms, and human bodies can develop various mechanisms to respond to these extrinsic materials. Given the potential bioactivities of carrier materials, it will be wise to pay attention to the effect of biomaterial itself in evaluating new formulation products and determine whether the altered pharmacological activities are indeed the consequence of the modification of drug delivery or attributable to the additive/ synergistic effects of the carrier at least partly. It will be only when we understand the actual role of the carrier materials in the body that we can develop a new drug delivery system with a significant effect on the drug distribution. Moreover, mechanistic understanding of activities of biomaterials will provide a new opportunity to develop macromolecular therapeutics for applications that low molecular weight drugs have not addressed.

\section{ACKNOWLEDGMENTS}

The authors acknowledge the support of NSF DMR1056997, NIH R01 EB017791, and Intramural Research Program (Global RNAi Carrier Initiative) of Korea Institute of Science and Technology.

\section{REFERENCES}

1. Bajaj G, Van Alstine WG, Yeo Y. Zwitterionic chitosan derivative, a new biocompatible pharmaceutical excipient, prevents endotoxin-mediated cytokine release. PLoS One. 2012;7(1):e30899.

2. Cho EJ, Sun B, Doh KO, Wilson EM, Torregrosa-Allen S, Elzey $\mathrm{BD}$, et al. Intraperitoneal delivery of platinum with in-situ crosslinkable hyaluronic acid gel for local therapy of ovarian cancer. Biomaterials. 2015;37:312-9.

3. Kumar MNVR, Muzzarelli RAA, Muzzarelli C, Sashiwa H, Domb AJ. Chitosan chemistry and pharmaceutical perspectives. Chem Rev. 2004;104:6017-84.

4. Doh KO, Yeo Y. Application of polysaccharides for surface modification of nanomedicines. Ther Deliv. 2012;3(12):1447-56.

5. Ilium L. Chitosan and its use as a pharmaceutical excipient. Pharm Res. 1998;15(9):1326-31.

6. Baldrick P. The safety of chitosan as a pharmaceutical excipient. Regul Toxicol Pharmacol. 2010;56(3):290-9.

7. Rao SB, Sharma CP. Use of chitosan as a biomaterial: studies on its safety and hemostatic potential. J Biomed Mater Res. 1997;34(1):21-8.

8. Muzzarelli RA, Mattioli-Belmonte M, Pugnaloni A, Biagini G. Biochemistry, histology and clinical uses of chitins and chitosans in wound healing. EXS. 1999;87:251-64.

9. Kojima K, Okamoto Y, Kojima K, Miyatake K, Fujise H, Shigemasa Y, et al. Effects of chitin and chitosan on collagen synthesis in wound healing. J Vet Med Sci. 2004;66(12):1595-8.

10. Kong M, Chen XG, Xing K, Park HJ. Antimicrobial properties of chitosan and mode of action: a state of the art review. Int $\mathbf{J}$ Food Microbiol. 2010;144(1):51-63.

11. Machado RL, de Arruda EJ, Santana CC, Bueno SMA. Evaluation of a chitosan membrane for removal of endotoxin from human $\mathrm{IgG}$ solutions. Process Biochem. 2006;41(11):2252-7.

12. Li J, Shao Y, Chen Z, Cong R, Wang J, Liu X. Membrane cartridges for endotoxin removal from interferon preparations. J Chromatogr B Analyt Technol Biomed Life Sci. 2003;791(1-2):55-61.

13. Xu P, Bajaj G, Shugg T, Van Alstine WG, Yeo Y. Zwitterionic chitosan derivatives for $\mathrm{pH}$-sensitive stealth coating. Biomacromolecules. 2010;11(9):2352-8.

14. Kim JH, Kim YS, Kim S, Park JH, Kim K, Choi K, et al. Hydrophobically modified glycol chitosan nanoparticles as carriers for paclitaxel. J Control Release. 2006;111(1-2):228-34.

15. Hwang HY, Kim IS, Kwon IC, Kim YH. Tumor targetability and antitumor effect of docetaxel-loaded hydrophobically modified glycol chitosan nanoparticles. J Control Release. 2008;128(1):23-31.

16. Min KH, Park K, Kim YS, Bae SM, Lee S, Jo HG, et al. Hydrophobically modified glycol chitosan nanoparticlesencapsulated camptothecin enhance the drug stability and tumor targeting in cancer therapy. J Control Release. 2008;127(3):208-18.

17. Chong BF, Blank LM, McLaughlin R, Nielsen LK. Microbial hyaluronic acid production. Appl Microbiol Biotechnol. 2005;66(4):341-51.

18. Oh EJ, Park K, Kim KS, Kim J, Yang JA, Kong JH, et al. Target specific and long-acting delivery of protein, peptide, and nucleotide therapeutics using hyaluronic acid derivatives. J Control Release. 2010;141(1):2-12.

19. Aruffo A, Stamenkovic I, Melnick M, Underhill CB, Seed B. CD44 is the principal cell surface receptor for hyaluronate. Cell. 1990;61(7):1303-13.

20. Ghosh SC, Neslihan Alpay S, Klostergaard J. CD44: a validated target for improved delivery of cancer therapeutics. Expert Opin Ther Targets. 2012;16(7):635-50.

21. Platt VM, Szoka Jr FC. Anticancer therapeutics: targeting macromolecules and nanocarriers to hyaluronan or CD44, a hyaluronan receptor. Mol Pharm. 2008;5(4):474-86.

22. Prestwich GD, Marecak DM, Marecek JF, Vercruysse KP, Ziebell MR. Controlled chemical modification of hyaluronic acid: synthesis, applications, and biodegradation of hydrazide derivatives. J Control Release. 1998;53(1-3):93-103.

23. Laurent TC, Laurent UB, Fraser JR. The structure and function of hyaluronan: an overview. Immunol Cell Biol. 1996;74(2):A1-7.

24. Fakhari A, Berkland C. Applications and emerging trends of hyaluronic acid in tissue engineering, as a dermal filler and in osteoarthritis treatment. Acta Biomater. 2013;9(7):7081-92.

25. Collins MN, Birkinshaw C. Hyaluronic acid based scaffolds for tissue engineering-a review. Carbohydr Polym. 2013;92(2):1262-79.

26. Tan B, Wang JH, Wu QD, Kirwan WO, Redmond HP. Sodium hyaluronate enhances colorectal tumour cell metastatic potential in vitro and in vivo. Br J Surg. 2001;88(2):246-50. 
27. Mytar B, Woloszyn M, Szatanek R, Baj-Krzyworzeka M, Siedlar $\mathrm{M}$, Ruggiero I, et al. Tumor cell-induced deactivation of human monocytes. J Leukoc Biol. 2003;74(6):1094-101.

28. Mytar B, Siedlar M, Woloszyn M, Colizzi V, Zembala M. Crosstalk between human monocytes and cancer cells during reactive oxygen intermediates generation: the essential role of hyaluronan. Int J Cancer. 2001;94(5):727-32.

29. Bajaj G, Kim MR, Mohammed SI, Yeo Y. Hyaluronic acid-based hydrogel for regional delivery of paclitaxel to intraperitoneal tumors. J Control Release. 2012;158(3):386-92.

30. Auzenne E, Ghosh SC, Khodadadian M, Rivera B, Farquhar D, Price RE, et al. Hyaluronic acid-paclitaxel: antitumor efficacy against CD44(+) human ovarian carcinoma xenografts. Neoplasia. 2007;9(6):479-86.

31. De Stefano I, Battaglia A, Zannoni GF, Prisco MG, Fattorossi A, Travaglia D, et al. Hyaluronic acid-paclitaxel: effects of intraperitoneal administration against $\mathrm{CD} 44(+)$ human ovarian cancer xenografts. Cancer Chemother Pharmacol. 2011;68(1):107-16.

32. Tomiyama T, Toita R, Kang JH, Koga H, Shiosaki S, Mori T, et al. Effect of introduction of chondroitin sulfate into polymerpeptide conjugate responding to intracellular signals. Nanoscale Res Lett. 2011;6(1):532.

33. Lee CT, Huang CP, Lee YD. Synthesis and characterizations of amphiphilic poly(L-lactide)-grafted chondroitin sulfate copolymer and its application as drug carrier. Biomol Eng. 2007;24(1):131-9.

34. Ranney D, Antich P, Dadey E, Mason R, Kulkarni P, Singh O, et al. Dermatan carriers for neovascular transport targeting, deep tumor penetration and improved therapy. J Control Release. 2005;109(1-3):222-35.

35. Lee CT, Huang CP, Lee YD. Preparation of amphiphilic poly(L-lactide)-graft-chondroitin sulfate copolymer selfaggregates and its aggregation behavior. Biomacromolecules. 2006;7(4):1179-86.

36. Park W, Park SJ, Na K. Potential of self-organizing nanogel with acetylated chondroitin sulfate as an anti-cancer drug carrier. Colloids Surf B: Biointerfaces. 2010;79(2):501-8.

37. Lim JJ, Hammoudi TM, Bratt-Leal AM, Hamilton SK, Kepple $\mathrm{KL}$, Bloodworth NC, et al. Development of nano- and microscale chondroitin sulfate particles for controlled growth factor delivery. Acta Biomater. 2011;7(3):986-95.

38. Liu YS, Chiu CC, Chen HY, Chen SH, Wang LF. Preparation of chondroitin sulfate-g-poly(epsilon-caprolactone) copolymers as a CD44-targeted vehicle for enhanced intracellular uptake. Mol Pharm. 2014;11(4):1164-75.

39. Fajardo AR, Guerry A, Britta EA, Nakamura CV, Muniz EC, Borsali R, et al. Sulfated glycosaminoglycan-based block copolymer: preparation of biocompatible chondroitin sulfate-bpoly(lactic acid) micelles. Biomacromolecules. 2014;15(7):2691700 .

40. Hamada K, Yoshihara C, Ito T, Tani K, Tagawa M, Sakuragawa $\mathrm{N}$, et al. Antitumor effect of chondroitin sulfate-coated ternary granulocyte macrophage-colony-stimulating factor plasmid complex for ovarian cancer. J Gene Med. 2012;14(2):120-7.

41. Pathak A, Kumar P, Chuttani K, Jain S, Mishra AK, Vyas SP, et al. Gene expression, biodistribution, and pharmacoscintigraphic evaluation of chondroitin sulfate-PEI nanoconstructs mediated tumor gene therapy. ACS Nano. 2009;3(6):1493-505.

42. Uchida S, Itaka K, Chen Q, Osada K, Miyata K, Ishii T, et al. Combination of chondroitin sulfate and polyplex micelles from Poly(ethylene glycol)-poly\{N - [N-(2-aminoethyl)-2aminoethyl]aspartamide\} block copolymer for prolonged in vivo gene transfection with reduced toxicity. J Control Release. 2011;155(2):296-302.

43. Hattori Y, Nakamura A, Arai S, Nishigaki M, Ohkura H, Kawano $\mathrm{K}$, et al. In vivo siRNA delivery system for targeting to the liver by poly-l-glutamic acid-coated lipoplex. Results Pharma Sci. 2014;4:1-7.

44. Imamura M, Kodama $\mathrm{Y}$, Higuchi N, Kanda K, Nakagawa $\mathrm{H}$, Muro T, et al. Ternary complex of plasmid DNA electrostatically assembled with polyamidoamine dendrimer and chondroitin sulfate for effective and secure gene delivery. Biol Pharm Bull. 2014;37(4):552-9.
45. Lo YL, Wang YS, Wang LF. The copolymer of poly(2dimethylaminoethyl methacrylate) and methacrylated chondroitin sulfate with low cytotoxicity for gene delivery. Adv Healthc Mater. 2013;2(11):1458-68.

46. Lo YL, Sung KH, Chiu CC, Wang LF. Chemically conjugating polyethylenimine with chondroitin sulfate to promote CD44mediated endocytosis for gene delivery. Mol Pharm. 2013;10(2):664-76.

47. Kurosaki T, Kitahara T, Kawakami S, Nishida K, Nakamura J, Teshima M, et al. The development of a gene vector electrostatically assembled with a polysaccharide capsule. Biomaterials. 2009;30(26):4427-34.

48. Naik RJ, Sharma R, Nisakar D, Purohit G, Ganguli M. Exogenous chondroitin sulfate glycosaminoglycan associate with arginine-rich peptide-DNA complexes to alter their intracellular processing and gene delivery efficiency. Biochim Biophys Acta. 2015;1848(4):1053-64.

49. Xu P, Quick G, Yeo Y. Gene delivery through the use of a hyaluronate-associated intracellularly degradable crosslinked polyethyleneimine. Biomaterials. 2009;30(29):583443.

50. Nikitovic D, Assouti M, Sifaki M, Katonis P, Krasagakis K, Karamanos NK, et al. Chondroitin sulfate and heparan sulfatecontaining proteoglycans are both partners and targets of basic fibroblast growth factor-mediated proliferation in human metastatic melanoma cell lines. Int J Biochem Cell Biol. 2008;40(1):72-83.

51. Mizumoto S, Sugahara K. Glycosaminoglycans are functional ligands for receptor for advanced glycation end-products in tumors. FEBS J. 2013;280(10):2462-70.

52. Trowbridge JM, Gallo RL. Dermatan sulfate: new functions from an old glycosaminoglycan. Glycobiology. 2002;12(9):117R$25 \mathrm{R}$.

53. Kabanov AV, Batrakova EV, Alakhov VY. Pluronic block copolymers for overcoming drug resistance in cancer. Adv Drug Deliv Rev. 2002;54(5):759-79.

54. Alakhov V, Klinski E, Li S, Pietrzynski G, Venne A, Batrakova $\mathrm{E}$, et al. Block copolymer-based formulation of doxorubicin. From cell screen to clinical trials. Colloids Surf B: Biointerfaces. 1999;16(1-4):113-34.

55. Zhao Y, Alakhova DY, Kabanov AV. Can nanomedicines kill cancer stem cells? Adv Drug Deliv Rev. 2013;65(13-14):1763-83.

56. Batrakova EV, Kabanov AV. Pluronic block copolymers: evolution of drug delivery concept from inert nanocarriers to biological response modifiers. J Control Release. 2008;130(2):98-106.

57. Alakhova DY, Rapoport NY, Batrakova EV, Timoshin AA, Li S, Nicholls D, et al. Differential metabolic responses to pluronic in MDR and non-MDR cells: a novel pathway for chemosensitization of drug resistant cancers. J Control Release. 2010;142(1):89-100.

58. Danson S, Ferry D, Alakhov V, Margison J, Kerr D, Jowle D, et al. Phase I dose escalation and pharmacokinetic study of pluronic polymer-bound doxorubicin (SP1049C) in patients with advanced cancer. Br J Cancer. 2004;90(11):2085-91.

59. Valle JW, Armstrong A, Newman C, Alakhov V, Pietrzynski G, Brewer J, et al. A phase 2 study of SP1049C, doxorubicin in Pglycoprotein-targeting pluronics, in patients with advanced adenocarcinoma of the esophagus and gastroesophageal junction. Invest New Drugs. 2011;29(5):1029-37.

60. Armstrong A, Brewer J, Newman C, Alakhov V, Pietrzynski G, Campbell S, Corrie P, Ranson M, and Valle JW. SP1049C as firstline therapy in advanced (inoperable or metastatic) adenocarcinoma of the oesophagus: a phase II window study. J Clin Oncol (Meeting Abstracts). 2006;24(18_suppl):4080.

61. Alakhova DY, Zhao Y, Li S, Kabanov AV. Effect of doxorubicin/ pluronic SP1049C on tumorigenicity, aggressiveness, DNA methylation and stem cell markers in murine leukemia. PLoS One. 2013;8(8):e72238.

62. Akbarzadeh A, Rezaei-Sadabady R, Davaran S, Joo SW, Zarghami N, Hanifehpour Y, et al. Liposome: classification, preparation, and applications. Nanoscale Res Lett. 2013;8(1):102.

63. Dams ET, Laverman P, Oyen WJ, Storm G, Scherphof GL, van Der Meer JW, et al. Accelerated blood clearance and altered 
biodistribution of repeated injections of sterically stabilized liposomes. J Pharmacol Exp Ther. 2000;292(3):1071-9.

64. Ishida T, Ichihara M, Wang X, Yamamoto K, Kimura J, Majima $\mathrm{E}$, et al. Injection of PEGylated liposomes in rats elicits PEGspecific IgM, which is responsible for rapid elimination of a second dose of PEGylated liposomes. J Control Release. 2006;112(1):15-25.

65. Lonez C, Vandenbranden M, Ruysschaert JM. Cationic liposomal lipids: from gene carriers to cell signaling. Prog Lipid Res. 2008;47(5):340-7.

66. David SA, Silverstein R, Amura CR, Kielian T, Morrison DC. Lipopolyamines: novel antiendotoxin compounds that reduce mortality in experimental sepsis caused by gram-negative bacteria. Antimicrob Agents Chemother. 1999;43(4):912-9.

67. Leon-Ponte M, Kirchhof MG, Sun T, Stephens T, Singh B, Sandhu S, et al. Polycationic lipids inhibit the pro-inflammatory response to LPS. Immunol Lett. 2005;96(1):73-83.

68. Wu Z, Nakanishi H. Phosphatidylserine-containing liposomes: potential pharmacological interventions against inflammatory and immune diseases through the production of prostaglandin E(2) after uptake by myeloid derived phagocytes. Arch Immunol Ther Exp (Warsz). 2011;59(3):195-201.

69. Ikehara Y, Yamanaka M, Yamaguchi T. Recent advancements in cytotoxic $\mathrm{T}$ lymphocyte generation methods using carbohydrate-coated liposomes. J Biomed Biotechnol. 2010;2010:242539.

70. Hosseini H, Li Y, Kanellakis P, Tay C, Cao A, Tipping P, Bobik A, Toh BH, Kyaw T. Phosphatidylserine liposomes mimic apoptotic cells to attenuate atherosclerosis by expanding polyreactive IgM producing B1a lymphocytes. Cardiovasc Res. 2015.

71. Ruvinov E, Harel-Adar T, Cohen S. Bioengineering the infarcted heart by applying bio-inspired materials. J Cardiovasc Transl Res. 2011;4(5):559-74.

72. Landesman-Milo D, Peer D. Altering the immune response with lipid-based nanoparticles. J Control Release. 2012;161(2):600-8.

73. Dodziuk H. Cyclodextrins and their complexes. WileyVCH, Weinheim. 2006.

74. Loukas YL, Vraka V, Gregoriadis G. Novel non-acidic formulations of haloperidol complexed with beta-cyclodextrin derivatives. J Pharm Biomed Anal. 1997;16(2):263-8.

75. Pourmokhtar M, Jacobson GA. Enhanced stability of sulfamethoxazole and trimethoprim against oxidation using hydroxypropyl-p-cyclodextrin. Pharmazie. 2005;60(11):837-9.

76. Kurkov SV, Loftsson T. Cyclodextrins. Int J Pharm. 2013;453(1):167-80.

77. Kiss T, Fenyvesi F, Bacskay I, Varadi J, Fenyvesi E, Ivanyi R, et al. Evaluation of the cytotoxicity of beta-cyclodextrin derivatives: evidence for the role of cholesterol extraction. Eur J Pharm Sci. 2010;40(4):376-80.

78. Hinzey AH, Kline MA, Kotha SR, Sliman SM, Butler ES, Shelton AB, et al. Choice of cyclodextrin for cellular cholesterol depletion for vascular endothelial cell lipid raft studies: cell membrane alterations, cytoskeletal reorganization and cytotoxicity. Indian J Biochem Biophys. 2012;49(5):329-41.

79. Hipler UC, Schonfelder U, Hipler C, Elsner P. Influence of cyclodextrins on the proliferation of $\mathrm{HaCaT}$ keratinocytes in vitro. J Biomed Mater Res A. 2007;83(1):70-9.

80. Qin L, Yang YB, Tuo QH, Zhu BY, Chen LX, Zhang L, et al. Effects and underlying mechanisms of curcumin on the proliferation of vascular smooth muscle cells induced by Chol:MbetaCD. Biochem Biophys Res Commun. 2009;379(2):277-82.

81. Portilho DM, Soares CP, Morrot A, Thiago LS, Butler-Browne $\mathrm{G}$, Savino $\mathrm{W}$, et al. Cholesterol depletion by methyl-betacyclodextrin enhances cell proliferation and increases the number of desmin-positive cells in myoblast cultures. Eur J Pharmacol. 2012;694(1-3):1-12.

82. Kopeček J, Kopečková P. HPMA copolymers: origins, early developments, present, and future. Adv Drug Deliver Rev. 2010;62(2):122-49.

83. Duncan R. Polymer conjugates as anticancer nanomedicines. Nat Rev Cancer. 2006;6(9):688-701.

84. Kopecek J. Polymer-drug conjugates: origins, progress to date and future directions. Adv Drug Deliv Rev. 2013;65(1):49-59.

85. Yang J, Kopecek J. Macromolecular therapeutics. J Control Release. 2014;190:288-303.

86. Wu K, Liu J, Johnson RN, Yang J, Kopecek J. Drug-free macromolecular therapeutics: induction of apoptosis by coiledcoil-mediated cross-linking of antigens on the cell surface. Angew Chem Int Ed Engl. 2010;49(8):1451-5.

87. Wu K, Yang J, Liu J, Kopecek J. Coiled-coil based drug-free macromolecular therapeutics: in vivo efficacy. J Control Release. 2012;157(1):126-31.

88. Kverka M, Hartley JM, Chu TW, Yang J, Heidchen R, Kopecek J. Immunogenicity of coiled-coil based drug-free macromolecular therapeutics. Biomaterials. 2014;35(22):588696.

89. Chu TW, Yang J, Zhang R, Sima M, Kopecek J. Cell surface selfassembly of hybrid nanoconjugates via oligonucleotide hybridization induces apoptosis. ACS Nano. 2014;8(1):719-30.

90. Chu TW, Kosak KM, Shami PJ, Kopecek J. Drug-free macromolecular therapeutics induce apoptosis of patient chronic lymphocytic leukemia cells. Drug Deliv Transl Res. 2014;4(56):389-94. 